\title{
Effects of DNA methyltransferase inhibition on pattern separation performance in mice
}

\author{
Anna Schlütter \\ Faculty of Health, Medicine and Life Sciences, Maastricht University \\ a.schluetter@student.maastrichtuniversity.nl
}

\begin{abstract}
Introduction: Schizophrenia and anxiety disorders place a great burden on the patients suffering from it and on society. The cognitive symptoms of both diseases can be caused partly by impaired pattern separation. Pattern separation is the ability to make distinct representations out of highly overlapping information. Improving pattern separation could slow down the progression of the above mentioned disorders and thus improve the quality of life of the patients. Alterations in gene expression can lead to differences in pattern separation performance. Methylation of DNA is one of the epigenetic changes controlled by DNA methyltransferases (DNMT) that can alter such gene expression levels without changing the underlying DNA sequence. In this study, the DNMT inhibitor RG108 was given to mice in order to investigate its effect on pattern separation performance.

Methods: 36 male $\mathrm{C}_{57} \mathrm{BL} / 6$ mice were used. Pattern separation performance was measured using the novel Object Pattern Separation task. It consists of two trials with two identical objects. In the second trial one of the objects is replaced along a vertical axis, while the second object stays in the same position. For the assessment of pattern separation the object is replaced at increasingly distinct locations. The most suitable inter-trial interval and position were assessed and pattern separation performance was measured after an intraperitoneal injection of either saline, $0.1 \mathrm{mg} / \mathrm{kg}$ RG108 or $0.3 \mathrm{mg} / \mathrm{kg}$ RG108. After 48 hours mice received a saline injection and pattern separation performance was measured again to check for carry-over effects. Lastly, a 24 hour inter-trial interval was used to unravel RG108 effects on long-term memory. After the behavioral task, the mice were decapitated and the dorsal hippocampus was dissected to quantify the expression of six target genes with qPCR.

Results: RG108 leads to an acute, dose-dependent increase in pattern separation. This effect vanishes after 48 hours. Furthermore, RG108 administration is not sufficient to
\end{abstract}


enhance pattern separation in such a way that animals can still remember the object's location after 24 hours. Whereas BDNF4, BDNF9, GRIA1, HDAC2 and HEY1 expressions do not change after acute administration of RG108, BDNF1 expression increases and can be the underlying reason for the improvement in pattern separation. Future studies are needed to reveal the possible methylation changes and to study the effect of chronic treatment with this drug.

\section{Keywords}

Anxiety disorder, BDNF, DNA methyltransferase inhibition, epigenetics, pattern separation, schizophrenia

\section{Introduction}

Schizophrenia and anxiety disorders have in common that they impose a great burden on the patients suffering from it and on society. In addition, the available treatment options fail to be very specific or only treat certain aspects of the disorder $(1,2)$. One brain process can be the underlying cause for the cognitive impairment of both disorders: Pattern separation in the hippocampus $(2,3)$. Pattern separation is the ability of forming distinct representations out of highly overlapping information (4). This is for example of importance for soldiers with a post-traumatic stress disorder (PTSD), who cannot distinguish between two similar situations: The gunshots in a threatening situation and fireworks in a safe situation (2). Enhancing pattern separation could be one way to help those people improving their quality of life. Enhancement of pattern separation requires changes in synaptic plasticity and changes in neuronal gene expression. Methylation of DNA is one way to change gene expression levels. It can decrease gene expression without changing the DNA sequence itself and is regulated by DNA methyltransferases (DNMT's) $(5,6)$.

To study the effect of DNA methylation on pattern separation performance, the DNMT inhibitor RG108 was given to mice before their pattern separation performance was measured with the novel Object Pattern Separation (OPS) task. Afterwards, gene expression of six target genes was measured in the dorsal hippocampus. The genes are important, either because they play a role in methylation of DNA or they are implicated in spatial learning and pattern separation. The expression of HDAC2, which de-acetylates DNA, was determined because it works in close concert with DNMT's and could therefore also be affected by DNMT inhibition (7). HEY1 in turn is responsive to Notch signaling, which is a crucial protein for neuronal development and plasticity $(8,9)$. GRIA1 belongs to the AMPAreceptor family. This family is crucial for long-term potentiation (LTP), the strengthening of synapses between nerve cells, which is important for learning and one form of synaptic 
plasticity (10). The different splice variants of BDNF are also implicated in early LTP (e-LTP), which lasts only one to two hours and late LTP (I-LTP), which lasts up to several days (11). Furthermore, BDNF is highly important for memory formation and learning and could therefore be an essential candidate in this task (12).

Improving pattern separation could be the first step towards the finding of new treatment options for schizophrenia and anxiety disorder patients. With this study, the effect of DNA methyltransferase inhibiton on pattern separation performance in mice should be unraveled. The aim of the qPCR study is to find gene expression changes that correlate with the behavior of the mice in the animal study.

\section{Material and methods}

The study was divided in three sub-experiments. The first part helped to assess the best object location and interval for the OPS task, which were subsequently used to determine the effect of different doses of the DNMT inhibitor RG108 on OPS performance. The third part included injection of RG108 at the same doses and thirty minutes after $\mathrm{T}_{1}$, the animals were decapitated and the RNA isolated from the dorsal hippocampus, in order to perform qPCR analysis of six target genes.

\section{Animals}

For this study, 36 three-months-old male $\mathrm{C}_{57} \mathrm{BL} / 6$ mice were used (Charles River Laboratories International, Inc., Sulzfeld, Germany) with an average weight of 24.1 grams at the beginning of the study. One mouse died prior to the experiment for unknown reasons. The mice were housed individually on sawdust bedding in standard Tecniplast IVC system greenline cage at $21^{\circ} \mathrm{C} \pm 2^{\circ} \mathrm{C}$, with a humidity of $50 \% \pm 10 \%$. The light/dark cycle was reversed, meaning that lights were off from 7:00 to 19:00 in order to perform the behavioral task in the active period of the mice under low illumination (20 lux). Furthermore, background noise by a radio was maintained to accustom the mice to noise. Food and water were available ad libitum. All experimental procedures were approved by the local ethics committee of Maastricht University for animal experiments and met governmental guidelines.

\section{Reagents}

RG108 was commercially obtained from Tocris Bioscience (Bristol, UK). The compound was dissolved in sterile physiological saline (B. Braun Melsungen AG, Melsungen, Germany) to produce an injection volume of $4 \mathrm{ml} / \mathrm{kg}$ of four different dilutions: $0.1 \mathrm{mg} / \mathrm{kg}, 0.3 \mathrm{mg} / \mathrm{kg}$, $1 \mathrm{mg} / \mathrm{kg}$ and $3 \mathrm{mg} / \mathrm{kg}$. The injection was given intraperitoneally thirty minutes before the first trial of the OPS task. 


\section{Object Pattern Separation task}

For the OPS task, a circular arena made of polyvinyl chloride (PVC) with a diameter and height of $40 \mathrm{~cm}$ was used. Half of the arena facing the experimenter was made of transparent PVC, whereas the other half was colored white. The objects used could not be moved by the mice. Two different types of objects were used in order to prevent familiarization, namely two massive aluminum cubes with tapering tops $(4.5 \mathrm{~cm} \times 4.5 \mathrm{~cm} \times 8.5 \mathrm{~cm})$, or two massive metal cubes $(2.5 \mathrm{~cm} \times 5 \mathrm{~cm} \times 7.5 \mathrm{~cm}$ ) with two holes (diameter $1.5 \mathrm{~cm}$ ).

The OPS task is divided in two trials ( $\mathrm{T}_{1}$ and $\mathrm{T}_{2}$ ) where animals are allowed to freely explore two identical objects. The objects are oriented in the same way during the whole experiment. Exploration was in this case defined as pointing the nose to the object at a distance of no more than $1 \mathrm{~cm}$ and/or touching the object. Sitting on, or leaning to, an object is not considered to be exploratory behavior. Before the start of the experiment, mice were handled daily for two weeks by the experimenter and were put in the arena to habituate them with the environment and the practices. Prior to the first and second trial, the natural exploration behavior of the mice was increased by placing them in an empty cage for four minutes.

After these four minutes, the mice were placed in the arena, facing the middle of the transparent half. They had four minutes time to explore the arena with the two identical objects on a horizontal line, approximately $5 \mathrm{~cm}$ from the wall (position 1; T1). After a predetermined interval ( $0.5 \mathrm{~h} / \mathrm{h} / 3 \mathrm{~h} / 4 \mathrm{~h}$ or $6 \mathrm{~h}$ ) in their home cage, the animals were put back into the arena for a second trial of four minutes. Now, one of the two objects was displaced along a vertical axis to one of four different distances from position 1 ( $T_{2}$ ). The left and right objects were randomly displaced to avoid bias because of place preferences of the animals. The time the animals spent exploring the objects during $\mathrm{T}_{1}$ and $\mathrm{T}_{2}$ was recorded and the amount of time spent on the stationary and on the moved object was calculated manually using a computer. Animals had to show sufficient exploration time in both trials, meaning that they were excluded from the study if their exploration time was below ten seconds.

After the OPS task, four direct measures are given: $\mathrm{a}_{1}, \mathrm{a}_{2}$, a3, and $\mathrm{b}$. A1 and a2 respectively indicate how much time the mice spent exploring each object location during $\mathrm{T} 1$, whereas as and $b$ respectively indicate the time spent on the stationary and the moved object in T2. From these four measures the variables e1, e2 and $d 2$ were calculated. The $d_{2}$ index, the measure for pattern separation performance, is a relative measure of discrimination corrected for exploratory activity and it can range from -1 to 1 . - 1 or 1 means complete preference for the familiar or novel object location, respectively, and o indicates no preference for either object location (13). 
For the first sub-experiment two training sessions were performed in order to find the best position for the objects and the best possible time interval between the first and second trial for the drug study. For this purpose, mice were randomly divided over one of the different positions in T2. Twelve mice were tested per position with a one hour interval, an interval in which mice are able to remember object locations (14). The position with the smallest distance to position $1 \mathrm{~L}$ or $1 \mathrm{R}$, at which the mice are able to distinguish the locations, was then used to determine the time interval in which mice are able to remember and detect spatial differences. For this second study, the mice were randomly divided over one of six different inter-trial intervals ( $0.5 \mathrm{~h} / 1 \mathrm{~h} / 2 \mathrm{~h} / 3 \mathrm{~h} / 4 \mathrm{~h} / 6 \mathrm{~h} ; \mathrm{N}=11-12$ ) and were tested on position 3 (13).

The best position and interval was then used to determine the effect of the DNMT inhibitor RG108 on pattern separation performance. Animals were equally divided in three experimental groups (vehicle, $0.1 \mathrm{mg} / \mathrm{kg}$ RG108, $0.3 \mathrm{mg} / \mathrm{kg} \mathrm{RG108)}$ and tested with a one hour interval at position three $(\mathrm{N}=11-12)$. Vehicle treatment was repeated in all animals after 48 hours to test a possible carry over effect. Finally, the animals were equally divided in five experimental groups (vehicle, $0.1 \mathrm{mg} / \mathrm{kg}$ RG108, 0.3 mg/kg RG108, $1 \mathrm{mg} / \mathrm{kg}$ RG108, 3 $\mathrm{mg} / \mathrm{kg}$ RG108) and tested with a 24 hour inter-trial interval at position three to investigate if an acute dose of RG108 has an effect on long-term memory.

For the last sub-experiments the animals were equally divided over the vehicle group, the $0.1 \mathrm{mg} / \mathrm{kg}$ RG108 group and the $0.3 \mathrm{mg} / \mathrm{kg}$ RG108 group. They were injected thirty minutes prior to the first trial of the OPS task ( $\left.\mathrm{T}_{1}\right)$. Thirty minutes after $\mathrm{T}_{1}$ the animals were decapitated and the hippocampus was dissected and frozen immediately.

\section{RNA isolation and CDNA synthesis}

RNA was isolated from the dorsal hippocampus samples using TRIzol ${ }^{\circledR}$ Reagent according to the manufacturer's protocol (Life Technologies, Carlsbad, California, USA) (15). The iScript $^{\text {TM }}$ CDNA Synthesis Kit was obtained from Bio-Rad Laboratories, Inc. (Hercules, California, USA) and cDNA was synthesized according to the manufacturer's protocol (16).

\section{qPCR}

The synthesized cDNA was used for TaqMan based qPCR assays. Gene expression was analyzed for $H D A C 2, B D N F 1,4$ and 9, HEY1, GRIA1 and three housekeeping genes (HPRT, B2M, $T B P$ ). The reaction mixture for each well in the 96 -wells plate contained $1 \mu$ l 20x TaqMan assay, 10 $\mathrm{l}$ 2 2x Taqman Universal Master Mix II with UNG (Applied Biosytems, Foster City, California, USA), $8 \mu$ I DEPC water and $1 \mu$ l of the respective CDNA ( $2 \mu l$ for BDNF9). All samples were pipetted and measured in duplo. 
All qPCR reactions were performed using the light cycler 480 (Roche, Basel, Switzerland) under the following conditions: One cycle of $50^{\circ} \mathrm{C}$ for two minutes to incubate UNG, one pre-incubation cycle of $95^{\circ} \mathrm{C}$ for ten minutes, 45 ( 55 for BDNF9) amplification cycles consisting of $95^{\circ} \mathrm{C}$ for fifteen seconds, $60^{\circ} \mathrm{C}$ for sixty seconds and $72^{\circ} \mathrm{C}$ for one second, and thirty seconds cooling at $40^{\circ} \mathrm{C}$. The following assays were used: $H D A C 2, M m 00515108$ m1; BDNF1, Mmo1334047_m1; BDNF4, Mm01334042_m1; BDNF9, Mmo4230564_m1; HEY1, Mmoo468865_m1; GRIA1, Mmoo433753_m1; HPRT, Mmoo446968_m1; B2M, Mmoo437762_m1 and TBP, Mmo0446973_m1. Prior to qPCR analysis, primer efficiency was checked with Standard cDNA (pooled from four samples, standard curve from 0.1 to $100 \mathrm{ng}$ (DNA) as well as the predicted amplicon length, which was checked by gelelectrophoresis. Two different software programs, Conversion_LC_480 and LinregPCR, were used to analyze the data.

\section{Statistics}

For the animal study one-sample Student's t-tests were performed in order to assess whether the $d 2$ index, for each experimental condition separately, differed significantly from zero. Animals which did not show sufficient exploration times were excluded (>10 seconds). Exploration times, different positions and different time intervals were compared using one-way ANOVAs. When the overall ANOVA was significant, LSD post-hoc analysis was performed. For the experiments with drug administration, one-way ANOVA with post-hoc Dunnett's test was performed. An a-level of 0.05 was considered significant. For the GPCR study, all samples were normalized with the value of the standard (10ong/ $\mathrm{ml}$ ) and corrected for the geomean of the three housekeeping genes. The different genes were then compared using one-way ANOVAs with a previous outlier correction. Outliers were tested for with Dixon's Q test $(17,18)$. When the overall ANOVA was significant, LSD post-hoc analysis was performed. An a-level of 0.05 was considered significant.

\section{Results}

Figure 1 depicts one of the training sessions with the OPS task, which measures the performance of the mice in the OPS task with five different positions of object 2 during T2 after a one hour interval. This training should help to assess the best possible position of object 2 during T2. The performance of the mice increases the further away the object is relocated during $T_{2} \quad\left(F_{4}, 53=3.302 ; P=0.017\right)$. Post-hoc analysis shows that memory performance at position 4 and 5 differs significantly from the control position $1(P=0.026$; $\mathrm{P}=0.002$ ) and position 2 and 3 differ significantly from position $5(\mathrm{P}=0.013 ; \mathrm{P}=0.048$; not shown). Furthermore, position 3 to 5 differ significantly from zero $(P=0.025 ; P=0.024$; 
$\mathrm{P}=0.001$; respectively). So, position 3 is the shortest distance between the objects in $\mathrm{T} 1$ and $\mathrm{T} 2$ where the animal still recognize that the object has been moved after a one hour interval. At this position, there is still room for improvement though since performance at positions 4 and 5 are significantly better.

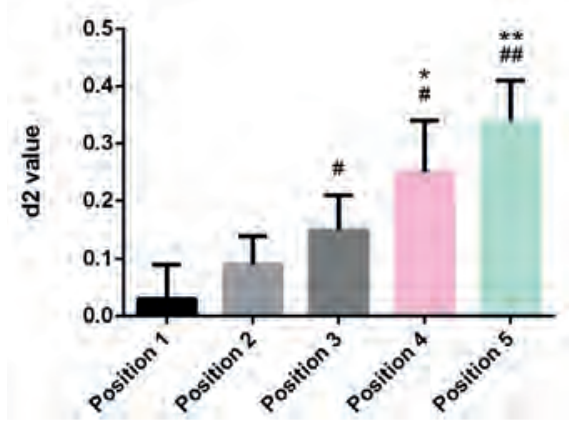

Figure 1. OPS performance on different positions: Object Pattern Separation performance (d2) after a 1 hour interval between $\mathrm{T}_{1}$ and $\mathrm{T}_{2}$ with different object positions. Data are shown as mean + SEM. A significant difference from zero is depicted with hashes (one sample t-tests, \#: $P<0.05$; \#\#: $\mathrm{P}<0.01$ ). A difference from position 1 is depicted with asterisks (one-way ANOVA followed by post-hoc LSD test, ${ }^{*}: \mathrm{P}<0.05 ;{ }^{* *}$ : $\mathrm{P}<0.01$ ). Positions 1-4, N = 12; Position 5, N=11.

Whereas a one-way ANOVA for e2 does not show any differences, e1 does show a significant difference $\left(F_{4}, 53=3.613 ; P=0.011\right)$. Post-hoc analysis reveals an increased exploration time with position 2 compared to the other positions ( $\mathrm{P}=0.019 ; \mathrm{P}=0.003 ; \mathrm{P}=0.006 ; \mathrm{P}=0.002$; data not shown).

The following training session was performed in order to find the most suitable interval for the OPS task with object 2 at position 3 . Figure 2 shows the $d 2$ value for different inter-trial intervals. The data shows that pattern separation performance is highest with an interval of 0.5 hours, as this interval shows the highest difference from zero ( $t$-test; $P \leq 0.001$ ). The one and two hour intervals also differ from zero ( $t$-test; $\mathrm{P}=0.025 ; \mathrm{P}=0.006$; respectively), so two hours is the longest interval where the animals still remember the object's location. One-way ANOVA shows a significant effect of interval duration on $d_{2}\left(\mathrm{~F}_{5}, 64=5.351 ; \mathrm{P} \leq\right.$ 0.001 ) and post-hoc analysis reveals that the smallest interval differs significantly from all other intervals (1h: $\mathrm{P}=0.010 ; 2 \mathrm{~h}: \mathrm{P}=0.022 ; 3 \mathrm{~h}: \mathrm{P}<0.001 ; 4 \mathrm{~h}: \mathrm{P}=0.001 ; 6 \mathrm{~h}: \mathrm{P}<0.001$ ). There were no significant differences in $\mathrm{e} 1$ and $\mathrm{e} 2$ between the different time intervals. 


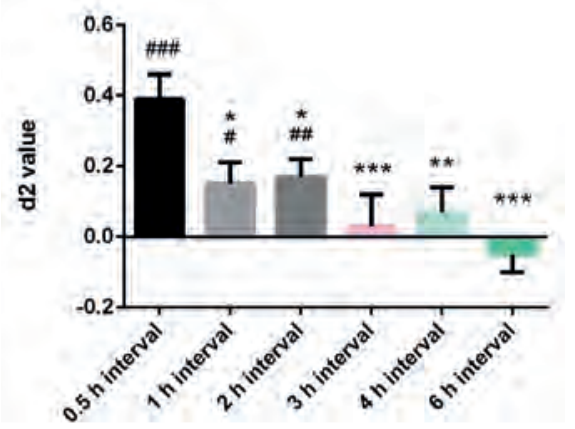

Figure 2. OPS performance on different inter-trial intervals: Object Pattern Separation performance (d2) at position 3 using different inter-trial intervals. Data are shown as mean + SEM. A significant difference from zero is depicted with hashes (one sample t-tests, \#: $\mathrm{P}<0.05$; \#\#: $\mathrm{P}<0.01$; \#\#\#: $\mathrm{P}<0.001$ ). A significant difference between $0.5 \mathrm{~h}$ and other time points is depicted with asterisks (one-way ANOVA followed by posthoc LSD test, $\left.{ }^{*}: \mathrm{P}<0.05{ }^{* *}: \mathrm{P}<0.01{ }^{* * *}: \mathrm{P}<0.001\right)$. $0.5-4 \mathrm{~h}$ intervals, $\mathrm{N}=12 ; 6 \mathrm{~h}$ interval, $\mathrm{N}=11$.

The object pattern separation performance at position 3 after a one hour inter-trial interval, and after injection of either saline, $0.1 \mathrm{mg} / \mathrm{kg}$ RG108 or $0.3 \mathrm{mg} / \mathrm{kg} \mathrm{RG108}$ thirty minutes before $\mathrm{T}_{1}$ is illustrated in Figure 3 . There are no differences in e1 and e2 between the three groups. The results of the t-tests are demonstrating that in all conditions $\mathrm{d} 2$ scores are significantly different from zero (saline: $\mathrm{P}=0.015 ; 0.1 \mathrm{mg} / \mathrm{kg}$ RG108: $\mathrm{P}=0.0001 ; 0.3 \mathrm{mg} / \mathrm{kg}$ RG108: $P<0.0001)$. The d2 value increases dose-dependently after administration of RG108. One-way ANOVA demonstrates a significant effect of drug treatment on d2 ( $F 2,32=6.188$; $\mathrm{P}=0.005$ ). Dunnett's post-hoc analysis revealed that a single injection with $0.3 \mathrm{mg} / \mathrm{kg}$ RG108 significantly improves memory performance compared to control ( $\mathrm{P}=0.003)$.

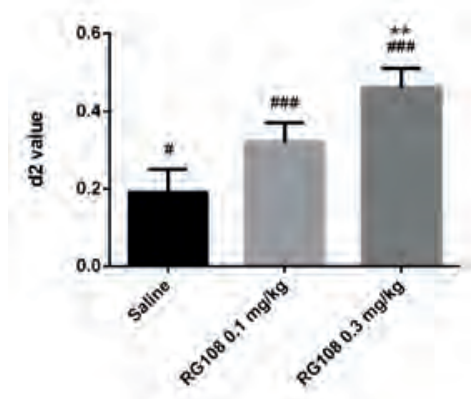

Figure 3. OPS performance after RG108treatment and $\mathbf{1}$ hour inter-trial interval: Object Pattern Separation performance ( $d_{2}$ ) at position 3 after a 1 hour interval between $T_{1}$ and $T_{2}$ and after RG108 treatment (30 minutes before $\mathrm{T} 1$ ). Data are shown as mean + SEM. A significant difference from zero is depicted with hashes (one sample t-tests, \#: P< 0.05; \#\#: P<0.01; \#\#\#: P<0.001). A significant difference from the saline condition is depicted with asterisks (one-way ANOVA followed by post-hoc Dunnett's test, ${ }^{* *}: \mathrm{P}<0.01$ ). RG108 treated groups, $\mathrm{N}=12$; saline group, $\mathrm{N}=11$. 
In addition to a first injection of either saline or RG108, all animals got an injection of saline 48 hours after the first injection to check for a possible carry-over effect (position 3, one hour interval). The results of the subsequent OPS tasks are displayed in Figure 4. E1 and e2 in all three groups do not differ significantly. Neither do the RG108 injected rats show a difference in $\mathrm{d} 2$ compared to the saline group. All groups remembered the object's location, as confirmed by t-tests (Saline: $\mathrm{P}=0.0001 ; 0.1 \mathrm{mg} / \mathrm{kg}$ RG108: $\mathrm{P}=0.0015 ; 0.3 \mathrm{mg} / \mathrm{kg}$ RG108: $P<0.0001)$.

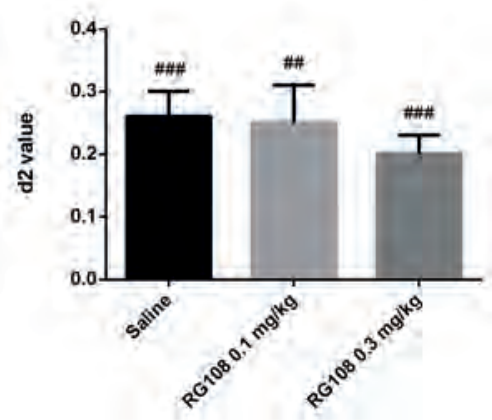

Figure 4. OPS performance after saline injection $48 \mathrm{~h}$ after RG108 treatment: Object Pattern Separation performance ( $\mathrm{d}_{2}$ ) at position 3 after a 1 hour interval between $\mathrm{T}_{1}$ and $\mathrm{T}_{2}$ and after a saline injection (i.p.) $48 \mathrm{~h}$ after the previous treatment. Data are shown as mean + SEM. A significant difference from zero is depicted with hashes (one sample t-tests, \#\#: P < 0.01; \#\#\#: P $<0.001$ ). No significant differences from the saline condition were found. $\mathrm{RG} 108$ treated groups, $\mathrm{N}=12$; saline group, $\mathrm{N}=11$.

In order to test whether higher doses of the compound could improve memory after a 24 hour interval, mice were again injected with either saline or one of four different concentrations of RG108 (0.1 mg/kg, $0.3 \mathrm{mg} / \mathrm{kg}, 1 \mathrm{mg} / \mathrm{kg}, 3 \mathrm{mg} / \mathrm{kg})$ and pattern separation performance was measured after a 24 hour interval (Figure 5). This was done in order to reveal changes in long-term memory after an acute dose of RG108. At this interval, animals did not remember the object's location and no differences in performance were found between the groups. 


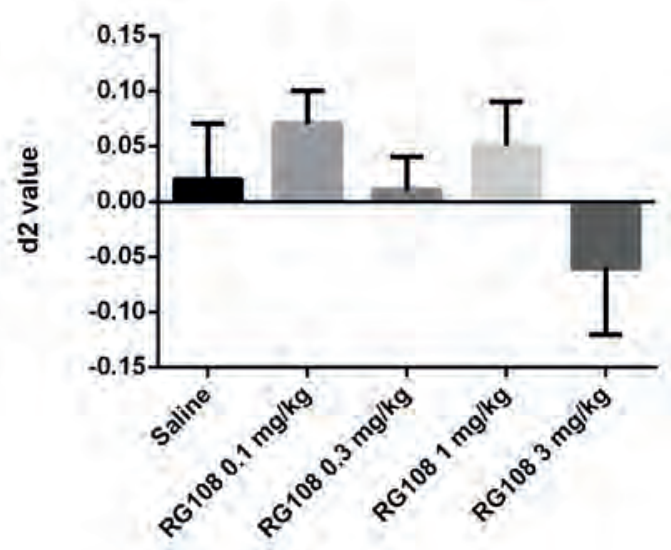

Figure 5. OPS performance after RG108 treatment and $\mathbf{2 4}$ hour inter-trial interval: Object Pattern Separation performance ( $\mathrm{d}_{2}$ ) at position 3 after a 24 hour interval between $\mathrm{T}_{1}$ and $\mathrm{T}_{2}$ and after RG10 8 treatment ( 30 minutes before $\mathrm{T} 1$ ). Data are shown as mean + SEM. No significant differences from zero or the saline condition were found. Saline, RG108 $0.3-3 \mathrm{mg} / \mathrm{kg}$ groups, $\mathrm{N}=12 ; \mathrm{RG} 1080.1 \mathrm{mg} / \mathrm{kg}$ group, $\mathrm{N}=11$.

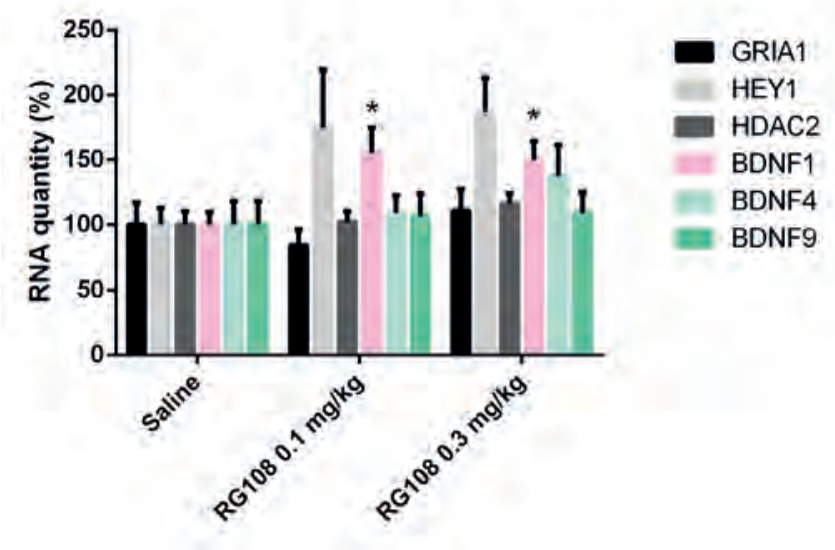

Figure 6. Expression level of target genes after RG108 treatment: Effects of RG108 $0.1 \mathrm{mg} / \mathrm{kg}$ and $0.3 \mathrm{mg} /$ kg i.p. compared to saline group on the expression level of GRIA1; HEY1; HDAC2; BDNF1; BDNF4 and BDNF9 in the mouse dorsal hippocampus. Values are percentages of the means + SEM compared to the saline group (corrected for geomean of TBP, B2M and HPRT). A significant difference from the saline condition is depicted with asterisks (one-way ANOVA followed by post-hoc LSD test, ${ }^{*}$ : P < 0.05 ). RG108 0.1 and $0.3 \mathrm{mg} / \mathrm{kg}$ groups, $\mathrm{N}$ $=12$; Saline group, $\mathrm{N}=11$. 
Approximately one month after the behavioral study, expression of GRIA1, HEY1, HDAC2, $B D N F 1, B D N F 4$ and BDNF9 was analyzed in the dorsal hippocampus by qPCR (Figure 6). The expression of the housekeeping genes was also analyzed. For the sake of clarity, gene expression was normalized to the respective saline condition. As expected, the housekeeping genes do not show any significant differences in mRNA expression. Whereas GRIA1, HEY1, HDAC2, BDNF4 and BDNF9 do not show significant differences in mRNA expression neither, treatment with $0.1 \mathrm{mg} / \mathrm{kg}$ RG108 and $0.3 \mathrm{mg} / \mathrm{kg}$ RG108 increased the expression of $B D N F 1$ compared to vehicle $(F 2,27=3,435 ; \mathrm{P}=0.047$; Dunnett's post-hoc: $\mathrm{P}=0.022 ; \mathrm{P}=0.042$; respectively).

\section{Discussion/Conclusion}

This study was performed to unravel the effect of RG108 on pattern separation performance in mice, and to find underlying gene changes that correlate with the findings of the animal study. For the behavioral OPS tasks position 3 was chosen to measure pattern separation. It is the intermediate position with room for improvement as well as room for a decline of pattern separation performance. In the first training session, mice showed an increased exploration time at position 2 . This seems to be an incidental finding as it is not seen in any of the other tasks, and can be caused by extensive behavioral testing (13). Exploration times were always sufficient (>10 seconds) and therefore all animals were included in the study. One hour was chosen as a time interval for drug testing, because mice show an intermediate performance at this interval. The 0.5 hour interval does show better pattern separation performance, but ceiling effects could hinder the interpretation of a pattern separation-improving drug. With a one hour interval performance can either be improved or it can decline and show a performance of a longer interval.

The administration of RG108 shows a dose-dependent improvement of pattern separation performance. The $0.3 \mathrm{mg} / \mathrm{kg}$ treated mice show a significantly better performance at the one hour interval than the saline treated mice. So, the inhibition of DNMT does indeed lead to an improvement in pattern separation in the mice. However, these changes in pattern separation performance are not long-lasting, as the OPS task 48 hours after the previous treatment does not show any change in pattern separation performance in the drug treated animals compared to the saline group. Furthermore, a 24 hour interval between trial one and two does not show any significant changes either, even if the dose is increased to $3 \mathrm{mg} / \mathrm{kg}$. This indicates that the acute administration of the DNMT inhibitor leads to an acute change in pattern separation performance, but the effect vanishes after 48 hours. Furthermore, administration of RG108 cannot increase the pattern separation performance in such a way that mice do still remember the object locations after a 24 
hour inter-trial interval. This suggests that no long-term plasticity changes occurred in the dorsal hippocampus. Further studies need to reveal whether the compound can increase pattern separation over a longer time interval if it is given chronically.

The qPCR analysis revealed that gene expression changes of the BDNF gene could explain the improved pattern separation performance of the mice. BDNF1 expression is upregulated by around fifty percent after injection of RG108. So, it is reasonable that the promoter of exon 1 gets de-methylated by the compound, therefore increasing expression. BDNF has many distinct roles in the CNS and is important for learning and memory, probably because of BDNF's role in LTP (19).

Considering the short time between injection, $\mathrm{T} 1$ and decapitation of the mice, it is unlikely that BDNF's role on I-LTP can explain the improved pattern separation in this experimental setup, because I-LTP sets in only two hours after induction of synaptic plasticity. However, BDNF is also important for e-LTP, which sets in directly and lasts for one to two hours and could therefore explain the acute improvement in pattern separation performance. LTP leads to a strengthening of stimulated synapses, which is crucial for learning (10). The mossy-fiber synapses of the hippocampus, which are the most relevant synapses for pattern separation performance (20), could represent a distinct type of e-LTP (nonassociative mossy fiber-LTP), which is independent of NMDA receptors. NMDA receptors play a crucial role in e-LTP in other synapses (11). The non-associative mossy fiber-LTP and BDNF's role in this type of LTP are not well understood and the exact mechanisms remain to be elucidated. The outcome of the qPCR study suggests that an increase in BDNF1 stimulates synaptic plasticity, so that the mice better remember the object's location.

In this study, $B D N F 1$ shows a significant increase in expression levels after DNMT inhibition, whereas BDNF4 and BDNFg do not show a change. This is possible because each exon of the BDNF gene has an independent promoter, of which some are preferentially targeted by epigenetic changes. It is surprising though that $B D N F_{4}$ is not affected by the DNMT inhibitor, as the promoter of the $B D N F_{4}$ exon is known to be susceptible to epigenetic changes (21). Further research is needed to reveal the exact roles of the different transcripts. What is known is that there is a region-specific distribution of certain variants in the central nervous system. Furthermore, different transcripts are involved in distinct cognitive tasks. In contrast to $\mathrm{BDNF}_{4}, \mathrm{BDNF}_{1}$ is also distributed in the dendrites of the neurons. Transcripts in the dendrites are associated with modulating changes linked to synaptic plasticity (21). The region-specificity, the task specificity as well as the susceptibility to epigenetic changes of the various BDNF transcripts can explain the differential response to the DNMT inhibitor RG108. A gene methylation array could unravel the methylation status of the BDNF gene and could therefore be used to gain more insights into the exact mechanisms of RG108 on the BDNF gene. 
Gene expression of GRIA1, HDAC2 and HEY1 are not altered by acute administration of RG108, despite their relevance for the task. HDAC2 is only linked to one of the two mechanism of methylation-induced gene silencing and could therefore remain unchanged even if methylation changes (5). It would be interesting to see whether there would also be no changes on mRNA level after chronic administration of RG108 and whether there are also no changes on the protein level. Posttranslational modifications cannot be examined with qPCR, but they can lead to changes on the protein level (22).

At first glance, HEY1 expression seems to be dose-dependently increased after RG108 administration although this did not reach statistical significance due to high standard deviations. The high standard deviation was found within the two different qPCR plates and the coefficient of variation for the individual duplos did not show any significant difference between the duplos. This suggests that $H_{E Y}$ expression has indeed a wide range of expression level in the mouse dorsal hippocampus. A methylation array would be useful to check whether DNMT inhibition affects the promoter of the gene.

In this study, the DNMT inhibitor RG108 was given intraperitoneally. Therefore, the inhibition could be distributed over the whole genome of the mice. This makes it necessary to check other brain regions than the dorsal hippocampus alone, as changes in those regions could also be the underlying cause for the observed behavior. Furthermore, such an approach could lead to many side-effects, as genome wide inhibition of methylation is associated with cancer metastasis, lupus or autoimmune disease (21). In the forebrain normal methylation is required for synaptic plasticity (5). It is therefore of utmost importance to check whether chronic treatment also improves pattern separation performance and does not show severe side effects.

This study gives first insights into the effect of epigenetic modifications on pattern separation. Inhibition of DNA methylation by acute administration of RG108 enhances pattern separation performance measured with the OPS task in a BDNF1-dependent manner. However, this effect is only acute, as it vanishes after 48 hours. Further studies are needed to reveal the exact change in DNA methylation and the effect of chronic administration of RG108. Furthermore, future studies have to show whether enhancement of pattern separation performance can help patients suffering from anxiety disorders, schizophrenia or AD.

\section{Role of the student}

Anna Schlütter was an undergraduate student in Biomedical Science working under the supervision of Dr.J.Prickaerts and Dr. ir.J. de Vry when the research in this report was performed. The topic was proposed by the supervisor. The qPCR study, the processing of the results as well as the formulation of the conclusions and the writing were done by the student. 


\section{Acknowledgments}

I would like to thank Dr. J. Prickaerts and Dr. ir. J. de Vry, who wrote and provided helpful comments on previous versions of the thesis and helped me during my practical work in the lab. Furthermore, I would like to thank N. P. van Goethem and B. T. J. van Hagen for their daily support while I was writing the thesis.

\section{References}

1. Schreiber R, Newman-Tancredi A. Improving cognition in schizophrenia with antipsychotics that elicit neurogenesis through 5-HT(1A) receptor activation. Neurobiology of Learning and Memory 110 (2014), 72-80. PubMed PMID: 24423786. Epub 2014/01/16. eng.

2. Kheirbek MA, Klemenhagen KC, Sahay A, Hen R. Neurogenesis and generalization: a new approach to stratify and treat anxiety disorders. Nature Neuroscience 15, 12 (2012), 1613-1620. PubMed PMID: 23187693 Pubmed Central PMCID: PMC3638121. Epub 2012/11/29. eng.

3. Tamminga CA, Stan AD, Wagner AD. The hippocampal formation in schizophrenia. The American Journal of Psychiatry 167, 10 (2010), 1178-1193. PubMed PMID: 20810471. Epub 2010/og/03. eng.

4. Clelland C, Choi M, Romberg C, Clemenson G, Fragniere A, Tyers P, et al. A functional role for adult hippocampal neurogenesis in spatial pattern separation. Science 325, 5937 (2009), 210-213.

5. Feng J, Fouse S, Fan G. Epigenetic regulation of neural gene expression and neuronal function. Pediatric research 61 (2007), 58R-63R.

6. Feng J, Zhou Y, Campbell SL, Le T, Li E, Sweatt JD, et al. Dnmt1 and Dnmt3a maintain DNA methylation and regulate synaptic function in adult forebrain neurons. Nature neuroscience 13, 4 (2010), 423-430.

7. Tsankova N, Renthal W, Kumar A, Nestler EJ. Epigenetic regulation in psychiatric disorders. Nature Review Neuroscience 8, 5 (2007):355-367. PubMed PMID: 17453016. Epub 2007/04/25. eng.

8. Maier MM, Gessler M. Comparative Analysis of the Human and Mouse $<\mathrm{i}\rangle$ Hey $1</ \mathrm{i}\rangle$ Promoter: $: \mathrm{i}\rangle \mathrm{Hey}\langle\mathrm{i}\rangle$ Genes Are New Notch Target Genes. Biochemical and Biophysical Research Communications 275, 2 (2000) 652-66o.

9. Costa RM, Honjo T, Silva AJ. Learning and memory deficits in Notch mutant mice. Current biology 13, 15 (2003), 1348-1354.

10. Lisman J, Yasuda R, Raghavachari S. Mechanisms of CaMKII action in long-term potentiation. Nature Review Neuroscience 13, 3 (2012), 169-182.

11. Edelmann E, Leßmann V, Brigadski T. Pre-and postsynaptic twists in BDNF secretion and action in synaptic plasticity. Neuropharmacology 76 (2014), 610-27.

12. Bekinschtein P, Oomen CA, Saksida LM, Bussey TJ, editors. Effects of environmental enrichment and voluntary exercise on neurogenesis, learning and memory, and pattern separation: BDNF as a critical variable? Seminars in Cell \& Developmental Biology (2011), Elsevier.

13. Van Hagen BTJ, van Goethem NP, Lagatta DC, Prickaerts J. The object pattern separation (OPS) task; a new behavioral paradigm derived from the object recognition task. Behavioral Brain Research, in press (2014).

14. Şık A, van Nieuwehuyzen P, Prickaerts J, Blokland A. Performance of different mouse strains in an object recognition task. Behavioral brain research 147, 1 (2003), 49-54.

15. Ambion. TRIzol ${ }^{\circledR}$ Reagent Carlsbad, CA, USA: Life Technologies; Downloaded from: https://www. lifetechnologies.com/order/catalog/product/15596026 (n.d.).

16. Bio-Rad Laboratories Inc. iScript ${ }^{T M}$ CDNA Synthesis Kit Hercules, CA, USA: Bio-Rad Laboratories Inc.; Downloaded from: http://www.bio-rad.com/en-us/product/reverse-transcription-reagents/iscript-cdnasynthesis-kit (n.d.). 
17. Dixon WJ. Analyses of extreme values. The Annals of Mathematical Statistics 21 (1959), 488-506.

18. Dixon WJ. Ratios involving extreme values. The Annals of Mathematical Statistics 22 (1959), 68-78.

19. Cunha C, Brambilla R, Thomas KL. A simple role for BDNF in learning and memory? Frontiers in molecular neuroscience 3, (2010).

20. Aimone JB, Deng W, Gage FH. Adult neurogenesis: integrating theories and separating functions. Trends in cognitive sciences 14, 7 (2010), 325-337.

21. Boulle F. Epigenetic regulation of BDNF/TrkB signaling in the pathophysiology and treatment of mood disorders [Dissertation]. Hertogenbosch, the Netherlands: Maastricht University and Université ParisDecartes; 2013.

22. Alberts B, Johnson A, Lewis J, Martin R, Roberts K, Walter P. Molecular Biology of the Cell. 5th ed. New York, USA: Garland Science; 2008. 